\title{
Two-dimensional surface state in the quantum limit of a topological insulator
}

\author{
James G. Analytis ${ }^{1,2 \star}$, Ross D. McDonald ${ }^{3}$, Scott C. Riggs ${ }^{4}$, Jiun-Haw Chu ${ }^{1,2}$, G. S. Boebinger ${ }^{4}$ \\ and Ian R. Fisher ${ }^{1,2}$
}

\begin{abstract}
The topological insulator is a unique state of matter that possesses a metallic surface state of massless particles known as Dirac fermions, which have coupled spin and momentum quantum numbers. Owing to the preservation of time-reversal symmetry, this coupling protects the wavefunctions against disorder ${ }^{1-3}$. The experimental realization of this state of matter in $\mathrm{Bi}_{2} \mathrm{Se}_{3}$ and $\mathrm{Bi}_{2} \mathrm{Te}_{3}$ has sparked considerable interest owing both to their potential use in spintronic devices and in the investigation of the fundamental nature of topologically nontrivial quantum matter. However, the conductivity of these compounds tends to be dominated by the bulk of the material because of chemical imperfection, making the transport properties of the surface nearly impossible to measure. We have systematically reduced the number of bulk carriers in $\mathrm{Bi}_{2} \mathrm{Se}_{3}$ to the point where a magnetic field can collapse them to their lowest Landau level. Beyond this field, known as the three-dimensional (3D) 'quantum limit', the signature of the 2D surface state can be seen. At still higher fields, we reach the 2D quantum limit of the surface Dirac fermions. In this limit we observe an altered phase of the oscillations, which is related to the peculiar nature of the Landau quantization of topological insulators at high field. Furthermore, we observe quantum oscillations corresponding to fractions of the Landau integers, suggesting that correlation effects can be observed in this new state of quantum matter.
\end{abstract}

The linear dispersion and energy quantization in field of the Dirac surface state of the archetypal topological insulators $\mathrm{Bi}_{2} \mathrm{Se}_{3}$ and $\mathrm{Bi}_{2} \mathrm{Te}_{3}$ have been revealed by angle-resolved photoemission spectroscopy and scanning tunnelling spectroscopy respectively ${ }^{4-9}$. However, the transport properties of the surface states, which are arguably their most technologically useful as well as fundamentally interesting characteristics, have proved to be especially difficult to measure owing to the overwhelming effect of bulk conduction channels ${ }^{10-14}$. The materials challenge is to find a way to cleanly eliminate the bulk conductivity so that the properties of the surface can be observed. By partially substituting ${ }^{15} \mathrm{Sb}$ for $\mathrm{Bi}$ in $\mathrm{Bi}_{2} \mathrm{Se}_{3}$ we systematically reduce the bulk carrier density to $n \sim 10^{16} \mathrm{~cm}^{-3}$. We study samples in which the Fermi surface of the Dirac fermion is small enough that at pulsed fields of up to $60 \mathrm{~T}$ we can access, for the first time, the 2D quantum limit (see Supplementary Information SA) - a regime where correlation effects are likely to arise ${ }^{16-20}$. In addition, we observe a distinguishing signature of topological insulators at high field whereby the phase of the oscillations is affected by the Zeeman energy.
In Fig. 1a we illustrate the dependence of the resistivity on temperature for representative single crystals with bulk carrier densities ranging from $\sim 10^{19} \mathrm{~cm}^{-3}$ down to $\sim 10^{16} \mathrm{~cm}^{-3}$. The carrier density was determined by the (low field) Hall effect, which matches the size of the Fermi surface measured by quantum oscillations, known as Shubnikov-de Haas oscillations (SdHO) (see Fig. 1b-d). These oscillations are periodic in inverse field and their period $\Upsilon$ can be related to the extremal cross-sectional area $A_{k}$ of the Fermi surface in momentum space through the Onsager relation $1 / \Upsilon=(\hbar / 2 \pi e) A_{k}$. When the field is rotated away from the crystal trigonal axis, the frequency of the $\mathrm{SdHO}$ changes according to the morphology of the Fermi surface. In every one of our samples, the bulk Fermi surface is a closed 3D ellipsoid ${ }^{21-23}$. The effective mass $m^{*} \sim 0.12 m_{\mathrm{e}}$ varies weakly with doping for these carrier densities, as previously reported ${ }^{21,22}$. For our lowest carrier density sample, the pocket is expected to have an orbitally averaged Fermi wavenumber of $k_{\mathrm{F}}=0.0095 \AA^{-1}$. This is small enough that we are able to exceed the bulk (3D) quantum limit with moderate fields $\sim 4 \mathrm{~T}$. This allows us to directly measure all of the relevant transport properties of the bulk, considerably simplifying our analysis, because there is no need to rely on surface-sensitive probes, which are inadequate for determining the bulk Fermi energy ${ }^{23}$. The remainder of this study is concerned with the $2 \mathrm{D}$ properties of the lowest carrier density samples at high fields.

In Fig. 2a,b we illustrate the longitudinal and transverse (Hall) resistances, $R_{x x}$ and $R_{x y}$ respectively, taken at $1.5 \mathrm{~K}$ on sample $\Sigma 1$ with $n \sim 4 \times 10^{16} \mathrm{~cm}^{-3}$. Strong features appear in $R_{x y}$ and $R_{x x}$ at similar fields in the bulk ultra-quantum limit ${ }^{24}$. To investigate the dimensionality of these features, we rotate the crystal in the field. For the 2D surface state of a topological insulator, quantum oscillatory phenomena depend only on the perpendicular component of the field $B_{\perp}$ and are periodic in $1 / B$. In Fig. $2 c$ we show the background-subtracted signal, illustrating that the oscillatory features grow with increasing field, as one might expect of SdHOs (for details of the background subtraction for all samples $\Sigma 1-3$, see Supplementary Information SC). In Fig. 2d,e we have plotted the $\mathrm{d} R_{x y} / \mathrm{d} B$ and $-\mathrm{d}^{2} R_{x x} / \mathrm{d} B^{2}$ as a function of $B_{\perp}=B \cos \theta$ - the usual procedure to determine whether minima in $R_{x x}$ (equivalent to minima in the negative second derivative) fall on top of the Hall plateaux (appearing as minima in the first derivative). Pronounced dips that are periodic in $1 / B$ in both the Hall derivative and the magnetoresistance align at all angles, providing unambiguous evidence that the plateau-like features in the Hall and minima in the SdHOs originate from a $2 \mathrm{D}$ metallic state.

\footnotetext{
${ }^{1}$ Stanford Institute for Materials and Energy Sciences, SLAC National Accelerator Laboratory, 2575 Sand Hill Road, Menlo Park, California 94025, USA, ${ }^{2}$ Geballe Laboratory for Advanced Materials and Department of Applied Physics Stanford University, California 94305, USA, ${ }^{3}$ Los Alamos National Laboratory, Los Alamos, New Mexico 87545, USA, ${ }^{4}$ National High Magnetic Field Laboratory and Department of Physics, 1800 E. Paul Dirac Drive, Florida State University, Tallahassee Florida 32310, USA. `e-mail: analytis@slac.stanford.edu.
} 
a

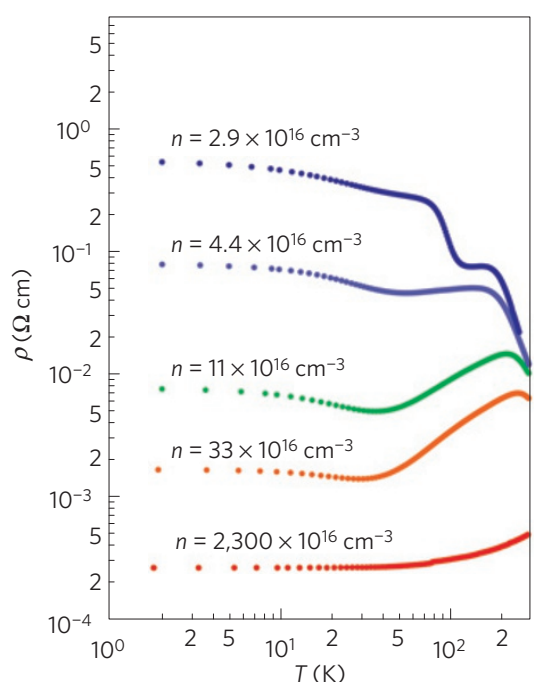

b

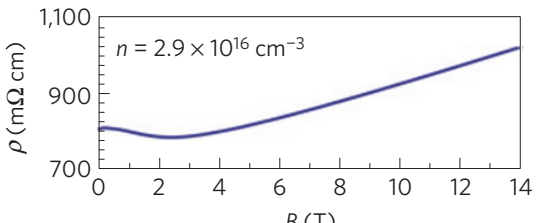

c

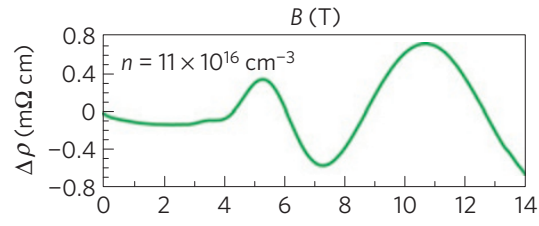

d

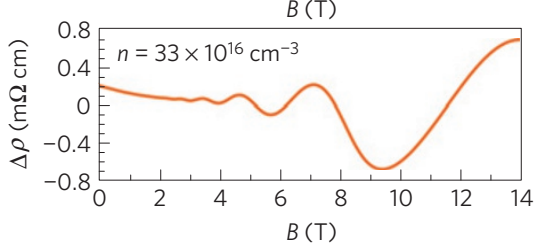

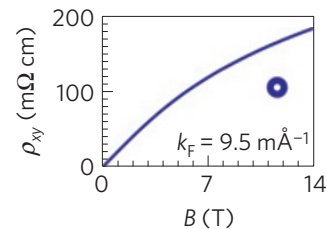

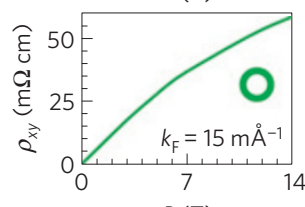

g

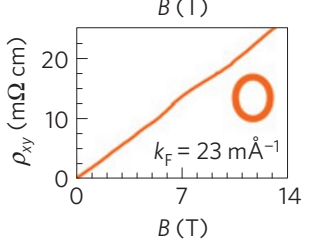

Figure 1 | Transport properties arising from the bulk Fermi surface. a, Progressively reducing the bulk carrier density in $\mathrm{Bi}_{2} \mathrm{Se}_{3}$ results in a significantly increased resistivity. Optimizing growth conditions for the binary melt can reduce carrier densities down to $n \sim 3 \times 10^{17} \mathrm{~cm}^{-3}$. Partial substitution of Sb for Bi allowed further reductions down to $n=2.9 \times 10^{16} \mathrm{~cm}^{-3}$. b-d, Representative magnetoresistance data for samples with indicated $n$, for fields oriented along the trigonal $c$ axis. The progressive reduction of the Fermi-surface volume is evident from the stretching of the SdHOs. $\mathbf{e - g}$, Carrier densities are extracted for each of these samples from the (low field) Hall effect. These agree with that expected from the size of the Fermi surface (shown schematically in the insets, with Fermi wave vector $k_{F}$ indicated). For the lowest carrier density samples shown in $\mathbf{e}$ and $\mathbf{f}$, the Hall signal deviates from linearity as the field approaches the 3D (bulk) quantum limit.

a

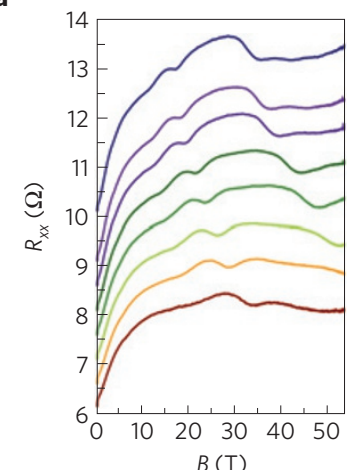

b

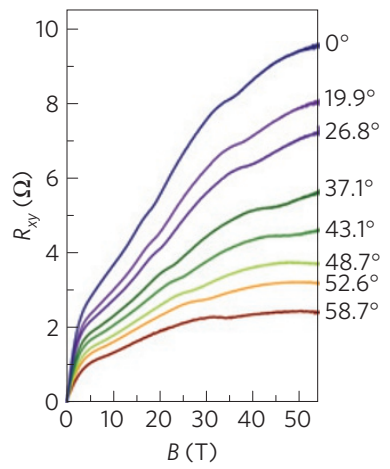

c

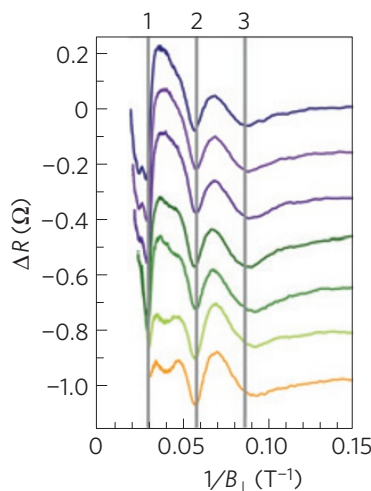

d

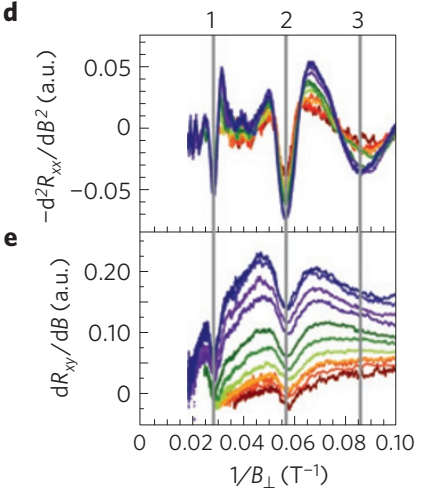

Figure 2 | Transport signature of the 2D surface state of a topological insulator. a, $\mathbf{b}, R_{x x}(\mathbf{a})$ and $R_{x y}(\mathbf{b})$ data traces as a function of magnetic field for indicated angles for a single crystal with $n \sim 4 \times 10^{16} \mathrm{~cm}^{-3}$ (sample $\Sigma 1$ ). The $R_{x x}$ traces are offset for clarity. The deviation from linearity of the low-field $R_{x y}$ indicates the 3D quantum limit $\left(B \sim 4 \mathrm{~T}\right.$ ), which does not vary with angle. Beyond this limit, additional features in both $R_{x y}$ and $R_{x x}$ move smoothly up in field as the tilt angle $\theta$ is increased. $\theta=0$ is defined to be when the field is perpendicular to the surface (parallel to the trigonal $c$ axis). $c$, A smooth second-third order polynomial can be fitted as background and subtracted from the raw $R_{x x}$, revealing oscillations that grow with field (see Supplementary Information SC). $\mathbf{d}, \mathbf{e},-\mathrm{d}^{2} R_{x x} / \mathrm{d} B^{2}(\mathbf{d})$ and $\mathrm{d} R_{x y} / \mathrm{d} B(\mathbf{e})$ as a function of $1 / B_{\perp}$, where $B_{\perp}=B \cos \theta$ aligns all features associated with the $2 D$ surface state. The vertical lines in $\mathbf{c}-\mathbf{e}$ indicate the first three Landau levels $N=1,2,3$ of the $2 \mathrm{D}$ state.

Examining Fig. 2c,d the second and third minima occur at twice and three times the $1 / B$ value of the first, and so we assign the Landau indices $N=1,2,3$ as shown. These indices connect a straight line through the origin when plotted versus $1 / B_{\perp}$ (Fig. 3b). The obvious candidate for the origin of the $2 \mathrm{D}$ states is the surface Dirac fermions. The plateau-like features in $R_{x y}$ are reminiscent of the quantum Hall effect in $2 \mathrm{D}$ electron gases ${ }^{24,25}$, but the quantization of the conductance is not evident (for a topological insulator this is expected to be $\sigma_{N}=2(N+1 / 2) e^{2} / h$; ref. 26). The discrepancy can be attributed to the remaining parallel conductance channel from the bulk described in detail below. Nevertheless, the periodicity and angular dependence of these data conclusively show that we are able to get to the quantum limit of the $2 \mathrm{D}$ state.

A crude method to confirm that the effect is surface-related is to briefly expose the sample to atmosphere, which is known to lead to $n$-type doping of the surface ${ }^{23}$. After $\sim 1 \mathrm{~h}$ exposure the oscillatory phenomena in Figs 2 and 3 are almost completely absent (see Supplementary Information SB and SH). Furthermore, if the origin of the signal is assumed to be a bulk $2 \mathrm{D}$ Fermi surface, the carrier contribution should be $\sim k_{\mathrm{F}}^{2} / 2 \pi c=6 \times 10^{18} \mathrm{~cm}^{-3}$, where $c=28.64 \AA$ is the lattice constant. Given that we can observe SdHO, such a pocket has a mobility large enough that it should dominate the Hall effect. Yet, we observe a carrier density 200 times smaller than this number and so a bulk origin of the $\mathrm{SdHO}$ seems unlikely. We conclude that the observed signal does indeed originate from the surface state of the topological insulator.

With this identification, the period $\Upsilon$ of the oscillations yields $k_{\mathrm{F}}=0.031 \AA^{-1}$ for the Dirac fermion, or $E_{\mathrm{F}} \approx 90 \mathrm{meV}$ (or $83 \mathrm{meV}$ when the Zeeman term is included, see below) above the Dirac point, using the value for the Fermi velocity ${ }^{23} v_{\mathrm{F}}=4.2 \times 10^{5} \mathrm{~ms}^{-1}$ 


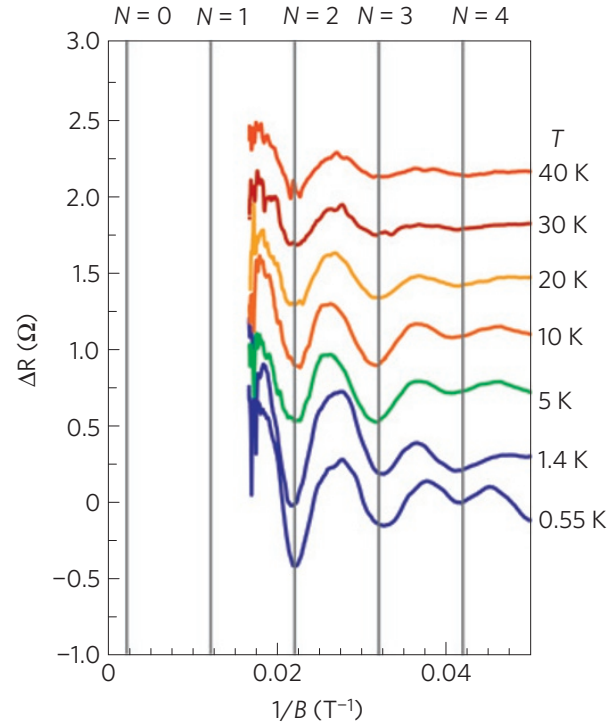

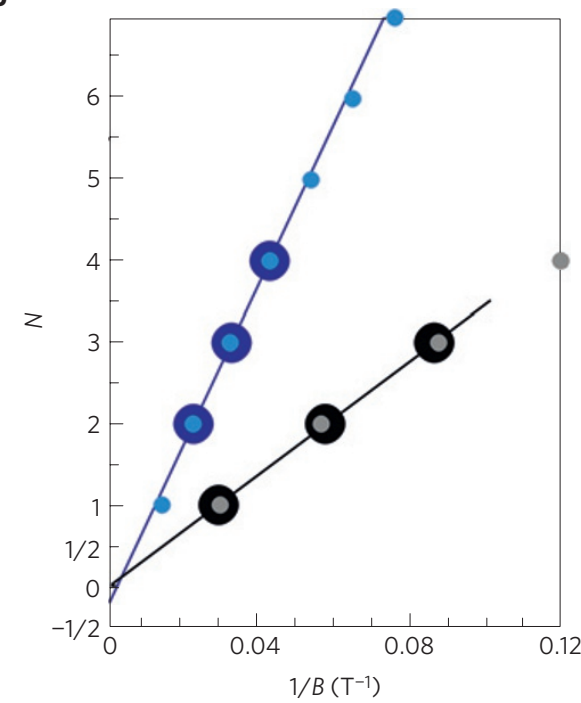

Figure 3 | Fermi energy dependence of the Landau intercept. a, Quantum oscillatory phenomena from sample $\Sigma 3$ from 0.55 to $40 \mathrm{~K}$. The higher Fermi energy leads to a larger slope and a reduced Landau intercept. b. The integer Landau levels from sample $\Sigma 1$ (black filled circles) and $\Sigma 3$ (dark blue filled circles) are shown as a function of inverse field. $\Sigma 1$ and $\Sigma 2$ have a zero intercept, which when fitted assuming a Landau quantized spectrum of $E_{N}^{ \pm}= \pm v_{F} \sqrt{2 N e \hbar B+\left(g_{s} \mu_{B} B / 2 v_{F}\right)^{2}}$ (see Supplementary Information SD) yields a $g$-factor $g_{\mathrm{s}} \approx 50$. The larger slope of $\Sigma 3$ makes the apparent intercept obtained from fitting $N=2$ to 4 negative, in quantitative agreement with our calculations (see Supplementary Information SD). The calculated values for the Landau intercept are shown in light blue and grey for the two different slopes, from fits to the observed Landau indices. The solid lines correspond to linear best fits to the experimental data. Note the Landau intercept does not correspond to what the filling factor is, which can be determined only by the quantized value of the Hall effect.

determined by photoemmission. This is consistent with the expected band bending ${ }^{23,27}$ at the surface for the given bulk carrier density (see Supplementary Information SA). Finally, a conventional Lifshitz-Kosevich analysis of the quantum oscillatory signal $^{28}$ (see Supplementary Information SG and SE) yields $k_{\mathrm{F}} l \approx 10$, where $k_{\mathrm{F}}$ is the Fermi wave vector and $l$ is the mean free path, and a surface mobility of $\mu=1,750 \mathrm{~cm}^{2} \mathrm{~V}^{-1} \mathrm{~s}^{-1}$.

It is important to distinguish this work from previous measurements of the Dirac fermion, which have been mostly done in zero field $^{4-7}$. The Zeeman term in the Hamiltonian, which determines spin coupling to the magnetic field, lifts the degeneracy at the Dirac point such that the quasiparticle gains a mass. This may account for the small mass enhancement we observe from the calculated zero-field cyclotron mass $\left(m_{2 \mathrm{D}}{ }^{*} \sim 0.089 m_{\mathrm{e}}\right.$ whereas we measure $0.11 m_{\mathrm{e}}$, see Supplementary Information SG). Furthermore, the Zeeman term can perturb the Landau quantization itself for a sufficiently large $g$-factor $g_{\text {s }}$ (see Supplementary Information SD) affecting the intercept of a plot of Landau index $N$ versus $1 / B$. If $g_{s}=0$, such a plot would yield an intercept of $(0,1 / 2)$. For $g_{\mathrm{s}} \neq 0$, the Landau index is weakly aperiodic and the last few Landau levels deviate from linearity yielding a smaller intercept $t^{20}$. For the period observed in our data, the observed intercept can be accounted for if $E_{\mathrm{F}}=83 \pm 5 \mathrm{meV}$ and $g_{\mathrm{s}} \approx 50 \pm 10$ (Fig. 3b). This estimation assumes the oscillations are sinusoidal, but a saw-tooth shape could reduce the $g$-factor considerably (see Supplementary Information SD). Nevertheless, $g_{\mathrm{s}} \approx 50$ is similar to reported bulk values ${ }^{29}$.

Notably, for a higher Fermi energy the intercept can seem to be negative if only the last few Landau levels are observed. In Fig. 3a we show data for sample $\Sigma 3$ with an SdHO frequency $F \approx 100 \mathrm{~T}$. Using a value of $g_{\mathrm{s}}=50$ obtained in the analysis of sample $\Sigma 1$ and fitting Landau level $N=2$ to $N=4$, one would expect an intercept of $(0,-0.17)$, in quantitative agreement with the observed value of $(0,-0.2)$, see Fig. $3 \mathrm{~b}$. This dependence of the apparent intercept on the Fermi energy and Landau indices fitted illustrates the peculiar relationship of the quantum oscillatory phase and the Zeeman term in the topological-insulator Hamiltonian, distinguishing this Dirac system from graphene $e^{30}$. The associated field dependence of the $N=0$ Landau level should in principle be observable through scanning tunnelling spectroscopy measurements. However, the anticipated shift in energy for a field of $10 \mathrm{~T}$ is only $16 \mathrm{meV}$, which is perhaps comparable to the tip drift between constant field traces, and thus eludes detection ${ }^{8,9}$.

To understand the amplitude of the oscillations we use a simple parallel resistor model to describe the effect of bulk and surface channels (see Supplementary Information SE). This analysis shows that the amplitude of the oscillations for $N \geq 2$ (for sample $\Sigma 2$ and $\Sigma 3$ ) is consistent with expectations if the Hall effect is assumed to be quantized in $\sigma_{x y}=2(N+1 / 2) e^{2} / h$ (see Supplementary Information $\mathrm{SE}$ and ref. 26). For sample $\Sigma 1$, a conservative estimate of $k_{\mathrm{F}} l \sim 1$ yields a (upper bound) discrepancy of a factor of approximately 20 at $N=1$. However, a more realistic estimate of $k_{\mathrm{F}} l$ will reduce this discrepancy considerably. This discrepancy might be partially due to difficulties in the background subtraction (see Supplementary Information SC), but more likely is related to inadequacies of the parallel resistor model. In particular, this model cannot account for surface-bulk coupling, inhomogeneities in the side surfaces or the anomalous nature of the topological-insulator Hall channel ${ }^{31,32}$.

Additional minima are visible in the second derivative $-\mathrm{d}^{2} R_{x x} / \mathrm{d} B^{2}$ versus $1 / B_{\perp}$ in the range $0<N<1$ and $1<N<2$, as shown in Fig. 4a,b. These approximately line up at each angle when plotted as a function of $1 / B_{\perp}$, and therefore also evidence $2 \mathrm{D}$ physics. These minima are not periodic in inverse field and thus cannot originate from another Fermi surface. They are also reproducible using different sweep rates of the pulsed magnetic field and thus cannot be artefacts of the pulsed magnet environment that are periodic in time (see Supplementary Information SF). Intriguingly, these minima fall very near simple rational fractions of the Landau integers $N=2 / 3,5 / 7$ and $4 / 5$ (in Fig. $4 \mathrm{a}$ ) as well as $N=6 / 5,4 / 3,3 / 2$ and 5/3 (in Fig. 4b). In Fig. 4c,d we show the background-subtracted data for $\Sigma 1$ and a further 


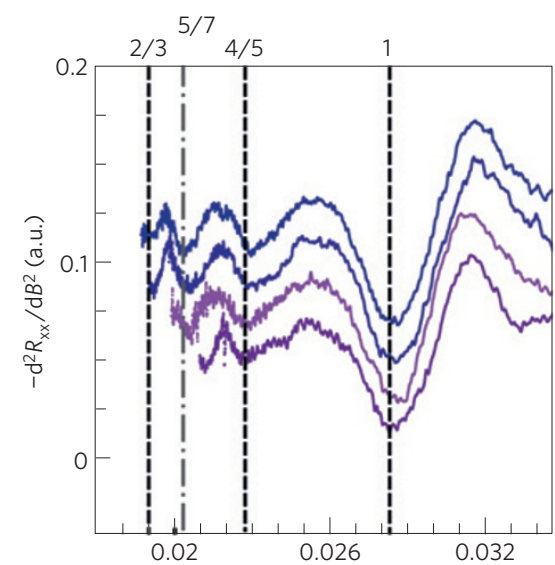

C

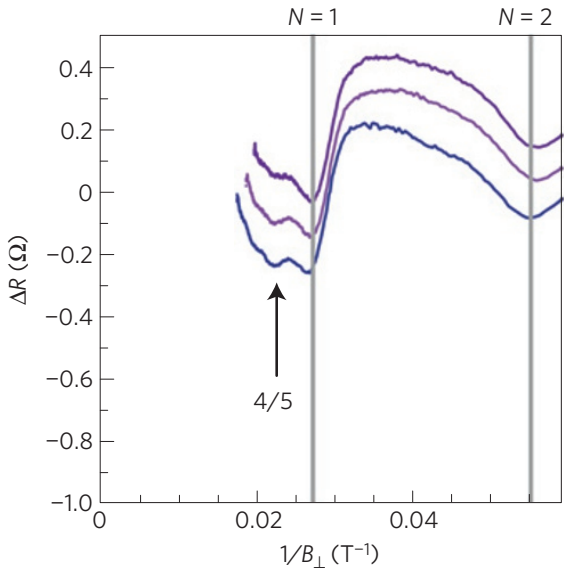

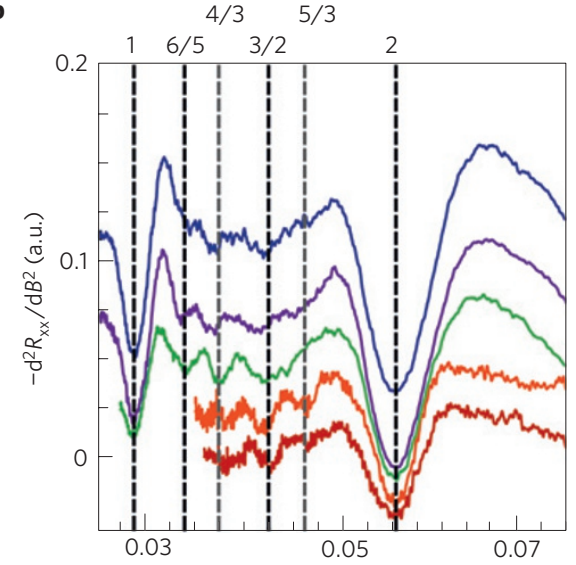

d

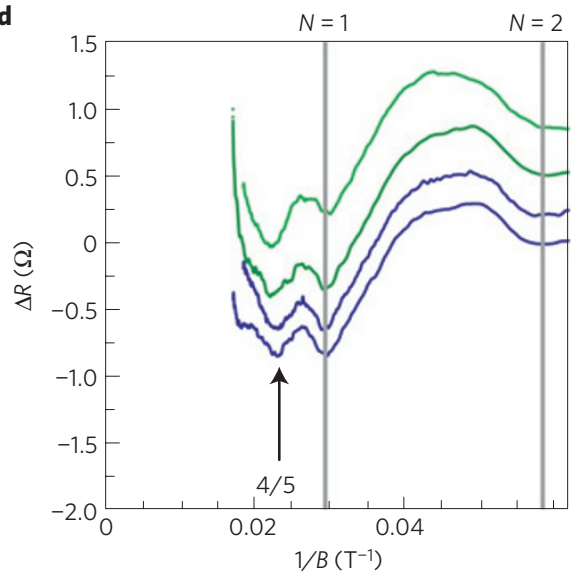

Figure 4 | Anomalous transport in the quantum limit of the 2D Fermi surface. $\mathbf{a}_{-}-\mathrm{d}^{2} R_{x x} / \mathrm{d} B^{2}$ as a function of $1 / B_{\perp}$ in the field range for which $N<1$ for $\theta=0^{\circ}, 9.1^{\circ}, 19.9^{\circ}$ and $26.8^{\circ}$ (top to bottom), illustrating three additional features at fields corresponding to fractional $N$. $\mathbf{b}$, The same signal for the field range for which $1<\mathrm{N}<2$ for angles $\theta=0^{\circ}, 19.9^{\circ}, 43.1^{\circ}, 55.7^{\circ}$ and $58.7^{\circ}$, illustrating four further features. Vertical lines are drawn in $\mathbf{a}$ and $\mathbf{b}$ corresponding to the nearest fractional N. c, Background-subtracted data for sample $\Sigma 1$ at three representative angles $\left(\theta=0^{\circ}, 19.9^{\circ}\right.$ and $27^{\circ}$ bottom to top) illustrating a pronounced feature at a fraction of 4/5. d. Similar background-subtracted data for sample $\Sigma 2$ shown at four temperatures $T=0.6,1.5,4.4$ and $6 \mathrm{~K}$ (bottom to top). The data in all panels have been offset for clarity.

sample $\Sigma 2$. The signal in both samples is almost identical and in particular, the first pronounced fraction $(4 / 5)$ in $\Sigma 1$ is also pronounced in $\Sigma 2$. The correspondence of these minima to simple fractions of the Landau indices is striking, and is suggestive of precursors to the fractional quantum Hall effect. Whether these features originate from fractional filling or indeed from other unanticipated physics of topological insulators remains to be seen.

Experimental access to the transport of the surface state of $\left.\mathrm{Bi}_{1-x} \mathrm{Sb}_{x}\right)_{2} \mathrm{Se}_{3}$ provides a new laboratory for studying topologically non-trivial quantum matter and perhaps the effects of correlations among Dirac fermions too ${ }^{16-18}$. Although the material realization of a topological insulator with a truly insulating bulk remains elusive, the present study illustrates that quantum effects associated with the surface states of these materials can be observed if the bulk conductivity is sufficiently suppressed.

\section{Methods}

Crystals with bulk carrier densities of $2,300 \times 10^{16} \mathrm{~cm}^{-3}$ and $33 \times 10^{16} \mathrm{~cm}^{-3}$ (red and orange curves in Fig. 1a) were obtained by slow cooling a binary melt with different $\mathrm{Bi} / \mathrm{Se}$ ratios. The principal origin of these relatively high carrier densities is Se deficiency and antisite defects. Samples with $n \leq 11 \times 10^{16} \mathrm{~cm}^{-3}$ were obtained by slow cooling a ternary melt containing progressively more $\mathrm{Sb}$. Although $\mathrm{Sb}$ is isovalent with $\mathrm{Bi}$, Sb substitution apparently acts to control the defect density in the bulk crystals, reducing the bulk carrier density. Samples with the lowest carrier density used in this study were grown from a melt with a nominal $\mathrm{Sb} / \mathrm{Bi} / \mathrm{Se}$ ratio of 7:52:130. This mixture was sealed in quartz under a partial pressure of argon. The mixture was heated to a temperature of $740{ }^{\circ} \mathrm{C}$ in $14 \mathrm{~h}$, held for $4 \mathrm{~h}$ then cooled over $50 \mathrm{~h}$ to $550^{\circ}$, and then annealed inside the quartz ampule for $80 \mathrm{~h}$. Before measurements were carried out, samples were cleaved on both sides with a scalpel blade in a dry atmosphere, mounted with contacts (using conductive silver epoxy) and pumped to $10^{-4} \mathrm{mbar}$ within $20 \mathrm{~min}$. The leads made contact along the sides of the crystal to maximize the contact with the bulk. All samples were measured using a standard four-probe configuration. Magnetoresistance and Hall effect measurements were carried out at the National High Magnetic Field Laboratory (Los Alamos) in the short-pulse $(\sim 10 \mathrm{~ms}$ rise time) $55 \mathrm{~T}$ magnet. Sweeps at both negative and positive field polarities were measured for all temperatures and angular positions. The data were then 'symmetrized' by combining the data from each field polarity to extract the components that are even in magnetic field (magnetoresistance, $R_{x x}$ ) and odd in magnetic field (Hall resistance, $R_{x y}$ ). The sample was rotated so that $\theta=90^{\circ}$ corresponded to field parallel to the current. The sample used for the angle dependence study had dimensions $\sim 0.63 \times 0.36 \times 0.15 \mathrm{~mm}^{3}$.

\section{Received 9 March 2010; accepted 25 October 2010;} published online 21 November 2010

\section{References}

1. Bernevig, B. A. \& Zhang, S. Quantum spin Hall effect. Phys. Rev. Lett. 96, 106802 (2006).

2. Teo, J. C. Y., Fu, L. \& Kane, C. L. Surface states and topological invariants in three-dimensional topological insulators: Application to $\mathrm{Bi}_{1-x} \mathrm{Sb}_{x}$. Phys. Rev. B 78, 045426 (2008)

3. Zhang, H. et al. Topological insulators in $\mathrm{Bi}_{2} \mathrm{Se}_{3}, \mathrm{Bi}_{2} \mathrm{Te}_{3}$ and $\mathrm{Sb}_{2} \mathrm{Te}_{3}$ with a single Dirac cone on the surface. Nature Phys. 5, 438-442 (2009). 
4. Hsieh, D. et al. Observation of unconventional quantum spin textures in topological insulators. Science 323, 919-922 (2009).

5. Chen, Y. L. et al. Experimental realization of a three-dimensional topological insulator, $\mathrm{Bi}_{2} \mathrm{Te}_{3}$. Science 325, 178-181 (2009).

6. Hsieh, D. et al. A tunable topological insulator in the spin helical Dirac transport regime. Nature 460, 1101-1105 (2009).

7. Alpichshev, Z. et al. STM imaging of electronic waves on the surface of $\mathrm{Bi}_{2} \mathrm{Te}_{3}$ : Topologically protected surface states and hexagonal warping effects. Phys. Rev. Lett. 104, 016401 (2010).

8. Cheng, P. et al. Landau quantization of topological surface states in $\mathrm{Bi}_{2} \mathrm{Se}_{3}$. Phys. Rev. Lett. 105, 076801 (2010).

9. Hanaguri, T., Igarashi, K., Kawamura, M., Takagi, H. \& Sasagawa, T. Momentum-resolved Landau-level spectroscopy of Dirac surface state in $\mathrm{Bi}_{2} \mathrm{Se}_{3}$. Phys. Rev. B 82, 081305 (2010).

10. Peng, H. et al. Aharonov-Bohm interference in topological insulator nanoribbons. Nature Mater. 9, 225-229 (2010).

11. Checkelsky, J. G. et al. Quantum interference in macroscopic crystals of nonmetallic $\mathrm{Bi}_{2} \mathrm{Se}_{3}$. Phys. Rev. Lett. 103, 246601 (2009).

12. Taskin, A. \& Ando, Y. Quantum oscillations in a topological insulator $\mathrm{Bi}_{1-x} \mathrm{Sb}_{x}$ Phys. Rev. B 80 (2009).

13. Qu, D., Hor, Y. S., Xiong, J., Cava, R. J. \& Ong, N. P. Quantum oscillations and Hall anomaly of surface states in the topological insulator $\mathrm{Bi}_{2} \mathrm{Te}_{3}$. Science 329, 821-824 (2010).

14. Butch, N. P. et al. Strong surface scattering in ultrahigh-mobility $\mathrm{Bi}_{2} \mathrm{Se}_{3}$ topological insulator crystals. Phys. Rev. B 81, 241301 (2010).

15. Kulbachinskii, V. A. et al. Conduction-band structure of $\mathrm{Bi}_{2-x} \mathrm{Sb}_{x} \mathrm{Se}_{3}$ mixed crystals by Shubnikov de Haas and cyclotron resonance measurements in high magnetic fields. Phys. Rev. B 59, 15733 (1999).

16. Seradjeh, B., Moore, J. E. \& Franz, M. Exciton condensation and charge fractionalization in a topological insulator film. Phys. Rev. Lett. 103, 066402 (2009).

17. Ran, Y., Yao, H. \& Vishwanath, A. Composite fermions and quantum Hall stripes on the topological insulator surface. Preprint at http://arxiv.org/abs/1003.0901 (2010).

18. Levin, M. \& Stern, A. Fractional topological insulators. Phys. Rev. Lett. 103, 196803 (2009).

19. Behnia, K., Balicas, L. \& Kopelevich, Y. Signatures of electron fractionalization in ultraquantum bismuth. Science 317, 1729-1731 (2007).

20. Seradjeh, B., Wu, J. \& Phillips, P. Signatures of surface states in bismuth at high magnetic fields. Phys. Rev. Lett. 103, 136803 (2009).

21. Kohler, H. \& Fabbicius, A. Galvanomagnetic properties of $\mathrm{Bi}_{2} \mathrm{Se}_{3}$ with free carrier densities below $5 \times 10^{17} \mathrm{~cm}^{-3}$. Phys. Status Solidi B 71, 487-496 (1975).

22. Kohler, H. Conduction band parameters of $\mathrm{Bi}_{2} \mathrm{Se}_{3}$ from Shubnikov-de Haas investigations. Phys. Status Solidi B 58, 91-100 (1973).
23. Analytis, J. G. et al. Bulk fermi surface coexistence with Dirac surface state in $\mathrm{Bi}_{2} \mathrm{Se}_{3}$ : A comparison of photoemission and Shubnikov de Haas measurements. Phys. Rev. B 81, 205407 (2010).

24. Sarma, S. D. \& Pinczuk, A. Perspectives in Quantum Hall Effects: Novel Quantum Liquids in Low-Dimensional Semiconductor Structures (Wiley-Interscience, 1996).

25. Novoselov, K. S. et al. Room-temperature quantum Hall effect in graphene. Science 315, 1379 (2007).

26. Lee, D. Surface states of topological insulators: The Dirac fermion in curved two-dimensional spaces. Phys. Rev. Lett. 103, 196804 (2009).

27. Wray, L. A. et al. Observation of topological order in a superconducting doped topological insulator. Nature Phys. advance online publication (2010).

28. Shoenberg, D. Magnetic Oscillations in Metals (Cambridge Univ. Press, 1984).

29. Kohler, H. \& Wochner, E. The g-factor of the conduction electrons in $\mathrm{Bi}_{2} \mathrm{Se}_{3}$. Phys. Status Solidi B 67, 665-675 (1975).

30. Novoselov, K. S. et al. Two-dimensional gas of massless Dirac fermions in graphene. Nature 438, 197-200 (2005).

31. Yu, R. et al. Quantized anomalous Hall effect in magnetic topological insulators. Science 329, 61-64 (2010).

32. Tse, W. \& MacDonald, A. H. Giant magneto-optical kerr effect and universal faraday effect in thin-film topological insulators. Phys. Rev. Lett. 105, 057401 (2010).

\section{Acknowledgements}

The authors would like to thank O. Vafek, D. Goldhaber-Gordon, J. Williams, S. Zhang, X. Qi, C-X Liu, J. Maciejko, Y. Chen, R. Mong and J. Moore for useful discussions.

F. Corredor assisted with the crystal growth. The NHMFL is supported by NSF Division of Materials Research through DMR-0654118. R.D.M. acknowledges support from the US DOE, Office of Basic Energy Sciences 'Science in 100 T' programme. This work is supported by the Department of Energy, Office of Basic Energy Sciences under contract DE-AC02-76SF00515.

\section{Author contributions}

J.G.A., R.D.M., J-H.C. and I.R.F. conceived the experiment of exploring the bulk quantum limit for surface signal. J.G.A., R.D.M. and S.C.R. carried out the experiments. J.G.A. grew the samples. All authors contributed to the interpretation and analysis of the data and to the writing of the manuscript.

\section{Additional information}

The authors declare no competing financial interests. Supplementary information accompanies this paper on www.nature.com/naturephysics. Reprints and permissions information is available online at http://npg.nature.com/reprintsandpermissions. Correspondence and requests for materials should be addressed to J.G.A. 